Published in final edited form as:

Arch Womens Ment Health. 2013 April ; 16(2): 87-92. doi:10.1007/s00737-012-0317-8.

\title{
Less depressed or less forthcoming? Self-report of depression symptoms in women preparing for in vitro fertilization
}

\author{
Adam M. Lewis, BA ${ }^{\mathrm{a}}$, Dawei Liu, PhD $^{\mathrm{b}}$, Scott P. Stuart, MD $^{\mathrm{c}}$, and Ginny Ryan, MD, MA ${ }^{\mathrm{d}}$ \\ ${ }^{\text {aDepartment }}$ of Psychological and Quantitative Foundations, University of lowa, 200 Hawkins \\ Drive, lowa City, IA 52242 \\ bDepartment of Biostatistics, University of lowa, 200 Hawkins Drive, lowa City, IA 52242 \\ 'Department of Psychiatry, University of lowa Carver College of Medicine, 200 Hawkins Drive, \\ lowa City, IA 52242
}

dDepartment of Obstetrics and Gynecology, University of lowa Carver College of Medicine, 200 Hawkins Drive, lowa City, IA 52242

\begin{abstract}
PURPOSE-While depression has been associated with infertility treatments, it is not routinely assessed in women prior to undergoing in vitro fertilization (IVF) treatment. Findings are mixed regarding the degree to which women report depression prior to IVF. The purpose of this study was to: 1) examine response profiles in women preparing for IVF, and 2) compare responses to those of postpartum, primary care, and general population groups.
\end{abstract}

METHODS-Female IVF patients ( $\mathrm{n}=321 ; 19-45$ years) completed the PHQ-9 at their first visit. Clinical, demographic characteristics, and incidence of major depressive disorder (MDD) and other depressive disorder (ODD) were examined. Overall score distributions of the IVF group were compared to those of local postpartum patients, and published primary care and general populations.

RESULTS-Demographic or clinical characteristics did not account for response differences within the IVF group. The IVF group had lower incidences of MDD and ODD than a PHQ-9 normative group. Women in the IVF group reported no depressive symptoms significantly more than postpartum, primary care, and general population groups.

CONCLUSIONS-Women preparing to undergo IVF report fewer symptoms of depression than multiple comparison groups. Specific quality of life measures may be needed to assess distress in this population.

\section{Keywords}

depression; infertility; screening; IVF

Corresponding Author: Ginny L Ryan, MD, MA, Division of Reproductive Endocrinology \& Infertility, Department of Obstetrics \& Gynecology, University of Iowa Hospitals \& Clinics, 31332 PFP, 200 Hawkins Drive, Iowa City, IA 52242, ginny-ryan@uiowa.edu, Telephone: 319-384-9170, Fax: 319-384-9367.

${ }^{a}$ Final clinical diagnosis of MDD requires ruling out bipolar disorder and depressive symptoms better explained by bereavement, a general medical condition, or medication.

Authors' roles: Lewis, Adam M: Study design, execution, analysis, manuscript drafting and critical discussion

Liu, Dawei: Analysis and manuscript drafting

Stuart, Scott P: Study design, analysis and critical discussion

Ryan, Ginny L: Study design, execution, analysis, manuscript drafting and critical discussion 
Infertility is estimated to affect $7.4 \%$ to $15 \%$ of men and women of reproductive age in the United States, with just over half of infertile couples seeking medical care (Chandra et al. 2005; Breyer et al. 2010; Boivine et al. 2007). Infertile individuals experience a number of psychosocial and economic treatment barriers. These barriers include public stigma associated with infertility and its treatments (Verhaak et al. 2007), high treatment costs with few states mandating full insurance coverage (Schmidt 2007), and emotional distress related to the diagnosis and often prolonged treatment rounds (Peterson and Eifert 2011). Infertility has also been shown to negatively impact couples (Peterson et al. 2003), and family and social networks (Newton et al. 1999), and is associated with increased risk for depression and anxiety (Fassino et al. 2002). Due to its prevalence and degree of impact, infertility is a societal burden with significant emotional and financial costs for affected individuals, their partners and families.

While clinicians often observe stress in patients undergoing in vitro fertilization (IVF) treatment, and infertility-related stress has been associated with anxiety and depression in the literature, research findings are mixed regarding the extent to which infertile women experience anxiety and depressive symptoms at any given time point during IVF (Peterson and Eifert 2011; Fassino et al. 2002; Greil 1997; Friedler et al. 2011). Some researchers have suggested that IVF is a source of stress for infertile couples, especially given the emotional, financial, and time investment couples make in these treatments (Fassino et al. 2002). For example, emotional distress has been found in couples who have not had prior infertility treatments, and especially after an unsuccessful treatment round or in cycles following the first attempt (Boivin et al. 1998; Boivin et al. 1995; Hammarberg et al. 2001; Slade et al. 1997; Ardenti et al. 1999; Newton et al. 1990). However, other researchers found no significant differences in depression, as measured by several psychological measures at various points in treatment, between IVF patients and individuals from the general population (Edelmann et al. 1994).

Some researchers have suggested that infertile individuals may feel pressure to appear more psychologically "healthy" prior to undergoing IVF, for fear that reporting symptoms of depression or anxiety will make them ineligible for treatment (Greil 1997). For example, in one study the authors noted that administering the measures pre-IVF may have increased social pressure to minimize their distress (Wichman et al. 2011). Whether IVF patients tend to under-report or have less distress during various phases of treatment is unclear, since many studies do not take into account when these measures are administered.

Physicians also do not routinely screen for depression or anxiety in IVF patients in order to determine if the research reflects the patients they encounter - despite the availability of a number of brief, easily administered self-report measures that have been used to screen for distress in other primary care settings, including the Patient Health Questionnaire (PHQ) and the Beck Depression Inventory (BDI) (Spitzer et al. 1999; Gilbody et al. 2007; Beck et al. 1996). Researchers and clinicians need to further examine how women respond to these measures prior to undergoing IVF in order to determine whether these measures warrant routine clinical use. The purpose of this study was to 1) examine response profiles in women preparing for IVF, and 2) compare their responses to those of postpartum women - a group with higher likelihood of having undergone past IVF treatments (Lee et al. 2011), and to primary care and general population groups.

\section{Materials and Methods}

\section{Participants}

A retrospective chart review identified women who made initial IVF visits to the University of Iowa Center for Advanced Reproductive Care between April, 2009 and December, 2010. 
419 women or couples were seen as new IVF patients at the program's two sites during that time. During those visits, the women were routinely administered a paper version of the PHQ-9 by a medical assistant or nurse. This was not introduced as a screening measure for depression or infertility-related distress. The administrators only stated that a physician would review the woman's responses to this questionnaire in order to make appropriate mental health referrals if necessary. Responses to all individual items on the PHQ-9 were collected. Through chart review, patient demographics, and diagnostic and treatment data were also obtained for all subjects. The University of Iowa Institutional Review Board approved this study.

Our PHQ-9 data were compared to initial screening results obtained as part of a previous epidemiological study on 7,860 postpartum women in 7 Iowa counties. Women who had given birth in the 6 months prior to January 2008 were recruited via State of Iowa birth records and the University of Iowa Women's Wellness and Counseling Service, and were screened over the phone using the PHQ-9. We also compared our PHQ-9 IVF data to normative data that were obtained from previously published studies of the diagnostic validity of the PHQ-9 for mental disorders in 3,000 primary care patients (Spitzer et al. 1999). Finally, we compared our PHQ-9 IVF data to general population PHQ data obtained from the publically available 2005-2006 National Health and Nutrition Examination Survey (NHANES) results. These data are collected by the National Center for Health Statistics (NCHS) of the Centers for Disease Control and Prevention to assess the general health and nutritional status of the U.S. population (Centers for Disease Control and Prevention, 2005).

\section{Patient Health Questionnaire-9 (PHQ-9)}

The PHQ-9 is a 9-item self-report measure originally designed to assess for the presence of major depressive disorder (MDD) and other depressive disorder (ODD - a subthreshold diagnosis of MDD) in a primary care setting. On a 0-3 Likert Scale, patients rate the frequency over the past 2 weeks of symptoms associated with 9 Diagnostic and Statistical Manual for Mental Disorders (DSM-IV) (American Psychiatric Association, 1994) criteria for MDD. The PHQ-9 can be scored to yield a diagnosis of MDD or ODD using an algorithm based on DSM-IV criteria, and as a depression severity rating based on raw scores categorized into score ranges. The possible score range is 0 to 27 , with higher scores indicating greater depressive symptom severity. Typical reported categories of score ranges with descriptors are: 0-4 (Minimal or no symptoms), 5-9 (mild depression), 10-14 (minor depression or dysthymic disorder), 15-19 (moderately severe MDD), and 20-27 (severe MDD). The PHQ-9 measure has been shown to be psychometrically sound in a number of clinical populations, including gynecology outpatients in whom the PHQ-9 demonstrated good sensitivity (73\%) and excellent specificity (98\%) for MDD (Spitzer et al. 1999; Spitzer et al. 2000). ${ }^{1}$

\section{Statistical analysis}

Statistical analysis was performed using SAS software (SAS Institute, Cary, NC). A chisquare test was used to compare frequency distributions among collapsed categorical groups of score ranges. Nonparametric analysis was conducted to make comparisons in the overall distributions. A Bonferroni correction was used to calculate a significant $\mathrm{p}$ value based on the number of comparisons that were made. A $P$ value of $<.05$ was considered statistically significant.

\section{Results}

321 of the 419 patients completed the PHQ-9 screening tool, giving a response rate of $77 \%$. Table 1 shows the clinical characteristics of our IVF study group. There were no significant 
PHQ response differences within the IVF study group based on clinical characteristics, including duration of infertility, treatment history, infertility-related diagnosis, or whether infertility was primary or secondary, though there was a trend towards exclusive female infertility diagnosis being associated with higher scores $(P=.07)$. There were also no significant associations between PHQ responses and demographic characteristics including age, marital status, BMI, and race, within the IVF study group. Demographic data for the IVF study group and comparative groups can be found in Table 2.

Based on PHQ-9 diagnostic scoring, women in the IVF study group had significantly lower incidences of MDD ( $1 \%$ versus $10 \%, P<0.0001)$ and ODD $(2 \%$ versus $6 \%, P<0.01)$ than the sample of primary care patients used in the original publication of the measure (Spitzer et al. 1999). An initial run of raw score frequencies among the IVF study group indicated that 90\% of PHQ-9 scores fell within the 0-4 range (minimal or no symptoms). Few women (2\%) had scores greater than or equal to 10 , a range suggesting moderate to severe depressive symptoms. We decided to look further into the distribution of scores within the $0-4$ range and found that half of women (50\%) in that range had scores of 0 , indicating no depressive symptoms at all over the previous 2 weeks. In order to make meaningful population comparisons given the distribution of our data, scores of 0 were separated from conventionally used ranges, and higher scoring categories were collapsed together. This resulted in 4 categorical score groups of 0 (no depressive symptoms), 1-4 (mild), 5-9 (moderate), and $\geq 10$ (severe). Using these categories, we found that the overall distribution of scores for the IVF group was significantly different than both the distribution of NHANES general population scores and the scores from Iowa postpartum women $(P<.0001)$ (Table 3). There was a particularly notable difference between the number of women in the IVF group reporting no depressive symptoms and those reporting no symptoms in both comparison groups.

\section{Discussion}

Our study sought to examine depression self-report response profiles of women prior to undergoing IVF. This study was unique in its high response rate and the type of comparative groups we used. Our patients initiating IVF treatment rarely endorsed any significant depressive symptoms, and almost never screened positive for MDD. This was a statistically significant difference compared to several large comparison groups, including patients in primary care and postpartum settings, and a community sample. Within our IVF study group, there were no significant response differences based on a woman's demographic or clinical characteristics, including type of infertility diagnosis, duration of infertility or history of infertility treatment. The trend we found toward having an exclusively female infertility diagnosis and higher PHQ scores may indicate an increased psychological burden for infertile women whose partners are fertile.

Most notably, IVF patients endorsed no depressive symptoms significantly more often than individuals in the general population or in a population of postpartum women in Iowa. These findings suggest that women are less depressed prior to undergoing IVF, potentially feeling optimistic about improved chances to conceive. However, this low level of distress is inconsistent with many findings in the literature and with the anecdotal accounts by some clinicians (Peterson and Eifert 2011; Fassino et al. 2002; Greil 1997; Friedler et al. 2011). Further, researchers found that distraction-based strategies were effective ways for individuals anticipating a stressful medical procedure to cope, particularly suppressing emotions related to the procedure (Bennett et al., 2007; Fauerbach et al., 2002, Tschuschke et al., 2001). Perhaps women cope with their anticipatory distress related to IVF by suppressing their negative emotions. 
Nevertheless, some researchers have suggested that the IVF patient population may be more immune to distress or depression. Given the financial barriers to reproductive health care, and limited insurance options for IVF treatment specifically, patients who are diagnosed with infertility and receive IVF treatment tend to report higher incomes. However, researchers have found that socioeconomic status was not predictive of psychological distress and rates of depressive disorders (Kosidou et al. 2011; Anderson et al. 2009). Our sample also included more married individuals than found in typical age-matched populations, and marriage has been shown to be protective for depression in fertile couples; though, infertile couples often report strains in their relationships, and research on marital discord has been associated with increased risk for depression (Peterson et al. 2003; Whisman et al. 2006).

Having children is a widely shared value and major quality of life determinant across cultures (Lampic et al. 2006; Kisakye et al. 2010; Newport 2011). Quality of life, as defined by the World Health Organization, refers to "individuals' perception of their position in life in the context of the culture and value systems in which they live and in relation to their goals, expectations, standards and concerns" (The WHOQOL Group, 1995). Besides representing a failure to achieve a personal goal, infertility negatively impacts quality of life as it represents a failure to achieve a cultural value, and thus carries a degree of social stigma. This means that infertile individuals may face increased social pressures, including pressure to underreport other socially stigmatized conditions such as mental illness. The authors are thus cautious regarding a conclusion that women prior to undergoing IVF are less distressed than the general population.

It may also be the case that the PHQ is not the best measure of distress in women preparing for IVF. Recently, researchers developed and tested the first quality of life measure for use specifically with infertile men and women (Boivin et al, 2011). Potential items were generated through consultation with patients, individually and in focus groups, and with a multidisciplinary team of gynecologists, nurses, psychologists, and social workers. They identified 6 infertility-specific domains most impacting quality of life: anger, affection, fatigue, family understanding, patient interactions with clinicians, and quality of emotional services (Boivin et al. 2011). Further testing, development, and implementation of such specific psychological screening measures for this population may be needed to gauge the mental health needs of this population.

One limitation of our study was the small sample size given the distribution of scores - that is, the tendency of IVF responses to center around a score of 0 , and few IVF patients receiving PHQ-9 scores greater than 10. Thus, we did not have enough power to make population comparisons for this higher range of scores. We acknowledge that the national population samples used for comparison were not ideal given the age, gender, and demographic differences between the samples and the Iowa IVF and postpartum groups. Yet we believe, in fact, that our results might have been more robust had these variables been controlled for. This is due to the fact that the national population samples include more individuals least at risk for depression, such as older individuals and men, than the IVF sample. Rates of depression generally decline throughout adulthood, and some researchers have suggested old age is protective for depression (Jorm et al. 2005). Women are also twice as likely as men to receive a diagnosis of MDD (Weissman et al. 1996).

The emotional, social, and economic burden of infertility is one often misunderstood by unaffected individuals. As with many stigmatizing conditions, infertility is often a silent and isolating affliction. If infertility patients were routinely presented with screening measures more specific to their experiences, they may respond differently. Not only do psychological measures need to be administered routinely, and at successive treatment intervals, but they 
should also be used as an opportunity to normalize the distress infertile individuals may be experiencing. In order to provide optimal infertility care, clinicians need to ensure they are using the appropriate measures when necessary.

\section{Acknowledgments}

Funding

No financial support received

\section{References}

American Psychiatric Association. Diagnostic and statistical manual of mental disorders. 4. Washington, DC: 2000. text rev

Anderson I, Thielen K, Nygaard E, Diderichsen F. Social inequality in the prevalence of depressive disorders. J Epidemiol Commun H. 2009; 63:575-581.

Ardenti R, Campari C, Agazzi L, La Sala GB. Anxiety and perceptive functioning in infertile women during in-vitro fertilization: exploratory survey of an Italian sample. Hum Reprod. 1999; 143:126132.

Beck, A.; Steer, R.; Brown, G. Manual for the Beck Depression Inventory-II. 2. San Antonio, TX: Psychological Corporation; 1996.

Bennett C, Phelps K, Brain K, Hood JG. A randomized controlled trial of a brief self-help coping intervention designed to reduce distress when awaiting genetic risk information. J Psychosom Res. 2007; 63:59-64. [PubMed: 17586338]

Boivin J, Tafekaman JE, Tulandi T, Brender W. Reactions to infertility based on extent of treatment failure. Fertil Steril. 1995; 63:801-807. [PubMed: 7890066]

Boivin J, Andersson L, Skoog-Svanberg A, Hjelmstedt A, Collins A, Bergh T. Psychological reactions during in-vitro fertilization: similar response pattern in husbands and wives. Hum Reprod. 1998; 13:3262-3267. [PubMed: 9853892]

Boivin J, Bunting L, Collins JA, Nygren KG. International estimates of infertility prevalence and treatment-seeking: potential need and demand for infertility medical care. Hum Reprod. 2007; 22:1506-1512. [PubMed: 17376819]

Boivin J, Takefman J, Braverman A. The fertility quality of life (FertiQoL) tool: development and general psychometric properties. Hum Reprod. 2011; 26:2084-2091. [PubMed: 21665875]

Breyer BN, Smith JF, Shindel AW, Sharlip ID, Eisenberg ML. The impact of infertility on family size in the USA: data from the National Survey of Family Growth. Hum Reprod. 2010; 25:2360-2365. [PubMed: 20601679]

Centers for Disease Control and Prevention. national Health and Nutrition Examination Survey Questionnaire (NHANES). Hyattsville, MD: U.S. Department of Health and Human Services; 2005. National Center for Health Statistics. retrieved from http://www.cdc.gov/nchs/nhanes/ nhanes2005-2006/nhanes05_06.htm

Chandra A, Martinez GM, Mosher WD, Abma JC, Jones J. Fertility, family planning, and reproductive health of U.S. women: data from the 2002 National Survey of Family Growth. Vital Health Stat. 2005; 23:1-160.

Edelmann RJ, Connolly KJ, Bartlett H. Coping strategies and psychological adjustment of couples presenting for IVF. J Psychosom Res. 1994; 38:355-364. [PubMed: 8064653]

Fassino S, Piero A, Boggio S, Piccioni V, Garzaro L. Anxiety, depression and anger suppression in infertile couples: a controlled study. Hum Reprod. 2002; 17:2986-2994. [PubMed: 12407062]

Fauerbach J, Lawrence J, Haythornthwaite J, Richter L. Coping with the stress of a painful medical procedure. Behav Res Ther. 2002; 40:1003-1015. [PubMed: 12296486]

Friedler S, Glasser S, Azani L, Freedman LS, Raziel A, Strassburger D, Ron-El R, Lerner-Geva L. The effect of medical clowning on pregnancy rates after in vitro fertilization and embryo transfer.

Fertil Steril. 2011; 95:2127-2130. [PubMed: 21211796] 
Gilbody S, Richards D, Brealey S, Hewitt C. Screening for depression in medical settings with the Patient Health Questionnaire (PHQ): a diagnostic meta-analysis. J Gen Intern Med. 2007; 22:1596-1602. [PubMed: 17874169]

Greil AL. Infertility and psychological distress: a critical review of the literature. Soc Sci Med. 1997; 45:1679-1704. [PubMed: 9428088]

Hammarberg K, Astbury J, Baker HWG. Women's experience of IVF: a follow-up study. Hum Reprod. 2001; 16:374-383. [PubMed: 11157838]

Jorm AF, Windsor TD, Dear KBG, Antsey KJ, Christensen H, Rodgers B. Age group differences in psychological distress: the role of psychological risk factors that vary with age. Psychol Med. 2005; 35:1253-1263. [PubMed: 16168148]

Kisakye P, Akena WO, Kaye DK. Pregnancy decisions among HIV-positive pregnant women in Mulago hospital, Uganda. Culture, Health \& Sexuality. 2010; 12:445-454.

Kosidou K, Dalman C, Lundberg M, Hallqvist J, Isacsson G, Magnusson C. Socioeconomic status and risk of psychological distress and depression in the stockholm public health cohort: a populationbased study. J Affect Disorders. 2011; 134:160-167. [PubMed: 21665286]

Lampic C, Svanberg AS, Karlstrom P, Tyden T. Fertility awareness, intentions concerning childbearing, and attitudes towards parenthood among female and male academics. Hum Reprod. 2006; 21:558-564. [PubMed: 16293651]

Lee SH, Liu LC, Kuo PC, Lee MS. Postpartum depression and correlated factors in women who received in vitro fertilization treatment. J Midwifery Womens Health. 2011; 56:347-352. [PubMed: 21733105]

Newport, F. Desire to have children alive and well in america. Vol. 2011. The Gallup Poll; 2003.

Newton CR, Hearn MT, Yuzpe AA. Psychological assessment and follow-up after in vitro fertilization: assessing the impact of failure. Fertil Steril. 1990; 54:879-886. [PubMed: 2226921]

Newton CR, Sherrard W, Glavac I. The Fertility Problem Inventory: measuring perceived infertilityrelated stress. Fertil Steril. 1999; 72:54-62. [PubMed: 10428148]

Peterson BD, Eifert GH. Using acceptance and commitment therapy to treat infertility stress. Cognitive and Behavioral Practice. 2011; 18:577-587.

Peterson BD, Newton CR, Rosen KH. Examining congruence between partners' perceived infertilityrelated stress and its relationship to martial adjustment and depression in infertile couples. Family Process. 2003; 42:59-70. [PubMed: 12698599]

Schmidt L. Effects of infertility insurance mandates on fertility. J Health Econ. 2007; 26:431-446. [PubMed: 17129624]

Slade P, Emery J, Lieberman BA. A prospective, longitudinal study of emotions and relationship in invitro fertilization treatment. Hum Reprod. 1997; 12:183-190. [PubMed: 9043926]

Spitzer RL, Kroenke K, Williams JB. Validation and utility of a self-report version of PRIME-MD: the PHQ primary care study. Primary Care Evaluation of Mental Disorders. Patient Health Questionnaire. JAMA. 1999; 282:1737-1744. [PubMed: 10568646]

Spitzer RL, Williams JB, Kroenke K, Hornyak R, McMurray J. Validity and utility of the PRIME-MD patient health questionnaire in assessment of 3000 obstetric-gynecologic patients: the PRIME-MD Patient Health Questionnaire Obstetrics-Gynecology Study. Am J Obstet Gynecol. 2000; 183:759769. The World Health Organization Quality of Life assessment (WHOQOL): position paper from the World Health Organization. Soc Sci Med 1995;41:1403-1409. [PubMed: 10992206]

Tschuschke B, Hertenstein B, Arnold R, Bunjes D, Denzinger R, Kaechele H. Associations between coping and survival time of adult leukemia patients receiving allogeneic bone marrow transplantation: results of a prospective study. J Psychosom Res. 2001; 50:277-285. [PubMed: 11399286]

Verhaak CM, Smeenk JM, Evers AW, Kremer JA, Kraaimaat FW, Braat DD. Women's emotional adjustment to IVF: a systematic review of 25 years of research. Hum Reprod Update. 2007; 13:27-36. [PubMed: 16940360]

Weissman MM, Bland RC, Canino GJ, Faravelli C, Greenwald S, Hwu HG, Joyce PR, Karam EG, Chung-Kyoon L, Lellouch J, et al. Cross-national epidemiology of major depression and bipolar disorder. JAMA. 1996; 276:293-299. [PubMed: 8656541] 
Whisman MA, Weinstock LM, Tolejko N. Marriage and depression. Women and depression: a handbook for the social, behavioral, and biomedical sciences. 2006:219-240.

Wichman CL, Ehlers SL, Wichman SE, Weaver AL, Coddington C. Comparison of multiple psychological distress measures between men and women preparing for in vitro fertilization. Fertil Steril. 2011; 95:717-721. [PubMed: 21067728] 


\section{Table 1}

Clinical characteristics of the IVF group

\begin{tabular}{lc}
\hline (n=321) & \\
\hline Infertility Duration Mean, yrs (range) & $2.7(0-18)$ \\
Previous Infertility Treatment (\% yes) & 68 \\
Primary or Secondary Infertility (\%) & Primary (65) \\
Infertility Diagnosis (\%) & \\
Male & 38 \\
Ovulatory & 22 \\
Unexplained & 21 \\
Tubal & 17 \\
Female Age & 16 \\
Diminished Reserve & 14 \\
Endometriosis & 13 \\
Uterine & 4 \\
Recurrent pregnancy loss & 3.5 \\
Unknown & 3 \\
\hline
\end{tabular}




\section{Table 2}

Demographic characteristics of infertile women at the time of PHQ-9 screening relative to other samples

\begin{tabular}{lcccc}
\hline & IVF (n=321) & Primary Care (n=3000) & Postpartum (n=7860) & General Population (n=5334) \\
\hline Mean Age, yrs (range) & $33(19-45)$ & $46(18-99)$ & $29.7(18-49)$ & $45.2(18-85)$ \\
Gender (\%) & Female (100) & Female (66) & Female (100) & Female (52) \\
Marital Status (\%) & Married (95) & Married (48) & NR & Married (49) \\
Mean BMI (range) & $26(15-49)$ & $N R$ & $N R$ & $N R$ \\
Race/Ethnicity (\%) & & & & \\
White & 87 & 79 & 94 & 47 \\
Asian & 5 & $N R$ & 1.8 & $N R$ \\
Hispanic/Latino & 2 & 4 & 2.6 & 24 \\
Black & 1 & 13 & 2.4 & 24 \\
Other & 4 & $N R$ & $N R$ & 4 \\
No Response & 1 & $N R$ & $N R$ & 0 \\
\hline
\end{tabular}

NR - Data not reported 
Table 3

Distribution of PHQ-9 score ranges by population

\begin{tabular}{rcccc}
\hline Group & $\mathbf{0}$ & $\mathbf{1 - 4}$ & $\mathbf{5 - 9}$ & $>\mathbf{1 0}$ \\
\hline Iowa IVF $(\%)(\mathrm{n}=321)$ & $45.2^{* *}$ & 44.9 & 8.1 & 1.9 \\
NHANES $(\%)(\mathrm{n}=4836)$ & 34 & 44.5 & 14.7 & 6.8 \\
Postpartum women $(\%)(\mathrm{n}=7860)$ & 19.9 & 50.7 & 19.6 & 9.9 \\
\hline
\end{tabular}

*** The overall distributions of scores were significantly different from one another at a level of $P<.0001$ 